\title{
Enfermagem na redução das Infecções do Sítio Cirúrgico (ISC)
}

\author{
Nursing in the reduction of Surgical Site Infections (SSI) \\ Enfermería em la reducción de Infecciones en el Lugar Quirúrgico (ISQ)
}

\author{
Pedro Jorge da Silva Pires \\ ORCID: https://orcid.org/0000-0001-5331-6146 \\ Centro Universitário FAMETRO, Brasil \\ E-mail: pjsilva009@gmail.com \\ Suzan Luiza da Silva Pereira \\ ORCID: https://orcid.org/0000-0003-2007-531X \\ Centro Universitário FAMETRO, Brasil \\ E-mail: suzanluiza.luiz@gmail.com \\ Izabel Cruz da Rocha \\ ORCID: https://orcid.org/0000-0001-6379-685X \\ Centro Universitário FAMETRO, Brasil \\ E-mail: belzinha.marie@gmail.com \\ Graciana de Souza Lopes \\ ORCID: https://orcid.org/0000-0003-3615-9040 \\ Centro Universitário FAMETRO, Brasil \\ E-mail: gracilopess@hotmail.com
}

\begin{abstract}
Resumo
A Infecção em Sítio Cirúrgico (ISC) é definida como um processo infeccioso no qual acomete os tecidos, cavidades e órgãos abordados em processos cirúrgicos, sendo necessário um empenho especial para mantê-las sob controle, sendo denominadas como parâmetros de controle da qualidade no serviço prestado por uma instituição hospitalar. Objetivouse com o presente artigo descrever as condutas e gerenciamentos de enfermagem voltada para redução das infecções do sítio cirúrgico e a segurança do paciente na assistência de enfermagem. A revisão, após o processo de legibilidade e inelegibilidade, selecionou 12 artigos através de leituras exploratória, seletiva, analítica e interpretativa, provenientes das bases de dados LILACS e SCIELO. As pesquisas selecionadas nos proporcionaram um quantitativo de 320 trabalhos possíveis para o estudo desejado, sendo assim, ficaram 30 e após a leitura novamente destes resumos que estavam disponibilizados na íntegra e que, consequentemente foram relidos e novamente selecionados, chegou-se a um número de 12 estudos que chegaram no objetivo deste estudo de revisão. Com isso foi enfatizado sobre a necessidade de implementações de medidas educativas que alcancem todos os profissionais assistenciais que atuam nesse contexto, buscando não somente a conscientização, mas também o reconhecimento e a aplicação do conhecimento científico na prática profissional, fazendo disso um artifício fundamental no combate à infecção rotineira.
\end{abstract}

Palavras-chave: Infecção de ferida operatória; Enfermagem; Complicações no pós-operatório; Educação continuada.

\begin{abstract}
Surgical Site Infection (SSI) is defined as an infectious process in which it affects the tissues, cavities and organs covered in surgical procedures, requiring a special effort to keep them under control, being called quality control parameters in the service. provided by a hospital institution. The objective of this article was to describe the nursing conducts and management aimed at reducing surgical site infections and patient safety in nursing care. The review, after the readability and ineligibility process, selected 12 articles through exploratory, selective, analytical and interpretive reading, from the LILACS and SCIELO databases. The selected researches provided us with a quantity of 320 possible works for the desired study, thus, there were 30 and after reading again these abstracts that were available in full and that, consequently, were reread and selected again, a number of 12 studies that met the objective of this review study. Thus, it was emphasized the need for implementation of educational measures that reach all care professionals who work in this context, seeking not only awareness, but also the recognition and application of scientific knowledge in professional practice, making this a fundamental artifice in combat to routine infection.
\end{abstract}

Keywords: Operative wound infection; Nursing; Postoperative complications; Continuing education.

\section{Resumen}

La Infección del Sitio Quirúrgico (ISQ) se define como un proceso infeccioso en el cual afecta los tejidos, cavidades y órganos cubiertos en procedimientos quirúrgicos, requiriendo un esfuerzo especial para mantenerlos bajo control, 
llamándose parámetros de control de calidad en el servicio. institución. El objetivo de este artículo fue describir las conductas y el manejo de enfermería encaminadas a reducir las infecciones del sitio quirúrgico y la seguridad del paciente en el cuidado de enfermería. La revisión, luego del proceso de legibilidad e inelegibilidad, seleccionó 12 artículos mediante lectura exploratoria, selectiva, analítica e interpretativa, de las bases de datos LILACS y SCIELO. Las investigaciones seleccionadas nos proporcionaron una cantidad de 320 trabajos posibles para el estudio deseado, así, fueron 30 y luego de leer nuevamente estos resúmenes que estuvieron disponibles en su totalidad y que, en consecuencia, fueron releídos y seleccionados nuevamente, una cantidad de 12 estudios que cumplió el objetivo de este estudio de revisión. Así, se enfatizó la necesidad de implementar medidas educativas que lleguen a todos los profesionales del cuidado que laboran en este contexto, buscando no solo la sensibilización, sino también el reconocimiento y aplicación del conocimiento científico en la práctica profesional, haciendo de este un artificio fundamental en el combate a la rutina. infección.

Palabras clave: Infección de herida operatória; Enfermería; Complicaciones postoperatorias; Educación continua.

\section{Introdução}

A Infecção do Sítio Cirúrgico (ISC) é considerada uma das complicações possivelmente identificadas após procedimento cirúrgico, sendo estas definidas por atingir tecidos, órgãos e cavidades iniciando processo infeccioso na qual pode estar presente em todo e qualquer tipo de procedimento cirúrgico (OMS, 2016).

No ambiente hospitalar as ISC, acontecem em qualquer tipo de procedimento por menor invasivo que este seja, porém ainda assim está alta taxa de incidência ocorre mais em cirurgias potencialmente contaminadas, ou seja, aquelas realizadas em tecidos com a flora residente não numerosa ou onde a descontaminação é um processo difícil (Shi, 2014).

As ISC são consideradas uma das mais graves Infecções Relacionadas à Assistência à Saúde (IRAS), sendo assim as IRAS são definidas como qualquer infecção que foi obtida durante ou após a internação do paciente internado, definida como a principal causa do aumento da morbimortalidade, tempo de vida do paciente, tempo de tratamento e custos de assistência contínua. Como consequências desses problemas fez-se necessário e obrigatório a implementação da Comissão de Controle de Infecção Hospitalar (CCIH) (Rocha, et al., 2016).

O Centro Cirúrgico (CC) é um setor importante para a estrutura organizacional hospitalar, devido às diversidades de procedimentos prestados e recursos envolvidos, sendo estes em caráter eletivo quanto de emergência, tendo como objetivo a busca pela garantia na qualidade dos procedimentos, melhorado o acesso e economizando custos (Blanck, et al., 2015).

Tendo em vista que as intervenções invasivas no CC são consideradas como um cenário de grande risco, com atuação de diferentes profissionais, trabalhando de modo a reduzir eventos adversos (EA) relacionados a agravos perante a integridade física e consequentemente ao óbito (Sandes, et al., 2019).

A etiologia da ISC também se associa à uma série de fatores que potencializam o grau de contaminação, como o mau preparo do paciente no pré-operatório, seu estado clínico, grande tempo de espera no pré-operatório, demora do procedimento cirúrgico, antissepsia ineficiente no intra-operatório. Considerada de alta gravidade, as cirurgias ortopédicas, neurológicas e cardíacas destacam-se entre as ISC, pois envolvem materiais de implantes como próteses (Santos, et al., 2017).

Entre as estratégias utilizadas na diminuição do índice de ISC, destaca-se a redução da carga microbiana da pele do paciente que irá submeter-se ao procedimento cirúrgico, com uso de antissépticos, pois além da pele ser abarreira de proteção contra as agressões externas, abriga agentes patogênicos que pode causar a ISC (Franco, et al., 2017).

Com isso a técnica de antissepsia das mãos é considerada como um dos principais meios profiláticos e de controle das IRAS. De acordo com o National Instiute for Clinical Excellence (NICE), a equipe cirúrgica deve higienizar suas mãos antes de qualquer procedimento cirúrgico, usando uma escova descartável com solução antisséptica aquosa. Nas cirurgias subsequentes, as mãos devem ser higienizadas com soluções alcoólicas antes da colocação da luva estéril, devido a sua rápida velocidade de ação e atividade antimicrobiana (Batista, et al., 2019). 
A notificação e o acompanhamento durante a internação e na pós-alta hospitalar, assim como a vigilância realizada de forma padronizada no pré, intra e pós-operatório, mostram resultados significativos no controle e na redução de taxas de infecção (Severo, 2016).

A humanidade sempre foi acometida por tais complicações infecciosas, e muitas vezes os cientistas desconheciam as formas de contágio, as más condições de higiene e a inexistência de saneamento, permitiram as proliferações de doenças causadas por microrganismos, no qual se disseminavam rapidamente entre as pessoas enfermas, que com o tempo e o avanço tecnológico houve melhora da assistência a nível hospitalar (Griep, 2013).

No Brasil, a ISC predomina na terceira colocação entre as IRAS, abrangendo cerca de $14 \%$ a $16 \%$ daquelas vistas em pacientes internados. Segundo dados recentes publicados pela Sociedade Americana de epidemiologia Hospitalar (SHEA), nos EUA a ISC chega a comprometer cerca de $2 \%$ a $5 \%$ dos pacientes que internam para realização de processos cirúrgicos (Brasil, 2017).

Dessa maneira cerca de 77\% das mortes associadas por ISC, sendo que93\% delas geram infecção generalizada nos órgãos em que foi realizado o procedimento. No Brasil, em média, $11 \%$ das cirurgias resultam em algum tipo de infecção. As taxas de probabilidade no Brasil revelam algo em torno de $15 \%$ de acordo com a ANVISA, em que o paciente operado corre riscos de adquirir uma ISC (Brasil, 2017).

O enfermeiro deve identificar e avaliar as condições do paciente em um contexto geral, diminuindo seus medos e inseguranças em todo o processo cirúrgico, criando protocolos de intervenção de enfermagem, sendo assim, os cuidados enfermagem voltados ao paciente cirúrgico do pré-operatório até o pós-operatório, necessita da identificação dos fatores de risco internos e externos sendo estes imprescindíveis para a prevenção de possíveis complicações (Martins, et al., 2017).

Objetivou-se com o presente artigo descrever as condutas/gerenciamento de enfermagem voltadas para redução das infecções do sítio cirúrgico e a segurança do paciente na assistência de enfermagem.

\section{Metodologia}

Estudo exploratório, do tipo descritivo de Revisão Integrativa da Literatura (RIL), que possui a finalidade de reunir materiais bibliográficos com ampla abordagem metodológica, e através dos resultados obtidos, integrarem conceitos com a revisão de teorias, evidências e análise de problemas metodológicos (Souza, et al., 2020).

Foram utilizados artigos científicos das seguintes bases de bases: Biblioteca Científica Eletrônica Online (SCIELO) e Literatura Latino Americana e do Caribe em Ciências da Saúde (LILACS), mediante os seguintes descritores: "Infecção de ferida operatória" AND "Enfermagem” AND “Complicações no pós-operatório" AND "Educação Continuada".

Como critérios de elegibilidade foram selecionados artigos originais, disponibilizados gratuitamente, em língua portuguesa, publicados no período de 2012 a 2021, que tratam do tema pesquisado. Critérios de inelegibilidade foram: Artigos com texto incompleto, resumos, monografias, dissertações de mestrado, teses de doutorado e artigos com língua diferente do português.

Os artigos foram selecionados de acordo com os critérios de elegibilidade e inelegibilidade a partir dos com os títulos, posteriormente foi realizada a análise de resumos e finalmente os artigos foram lidos na integra, sendo elaborado um instrumento para a coleta de informações direto das bases de dados.

\section{Resultados}

As pesquisas nas bases de dados selecionadas nos proporcionaram um quantitativo de 320 trabalhos possíveis para o estudo desejado, depois de realizadas as leituras dos resumos/artigos, foram pré-selecionados quanto à sua relevância e à propriedade que permitiam responder aos objetivos propostos. 
Sendo assim, ficaram 30 trabalhos e após a leitura novamente destes resumos que estavam disponibilizados na íntegra e que, consequentemente foram relidos e novamente selecionados. Desses excluímos 18 que estavam repetidos em outras bases de dados. Chegou-se a um número de 12 estudos que atingiram o objetivo desta revisão de literatura e que foram preparados para compor a amostra da pesquisa. (Figura 1).

Figura 1: Fluxograma de seleção dos artigos para a RIL.

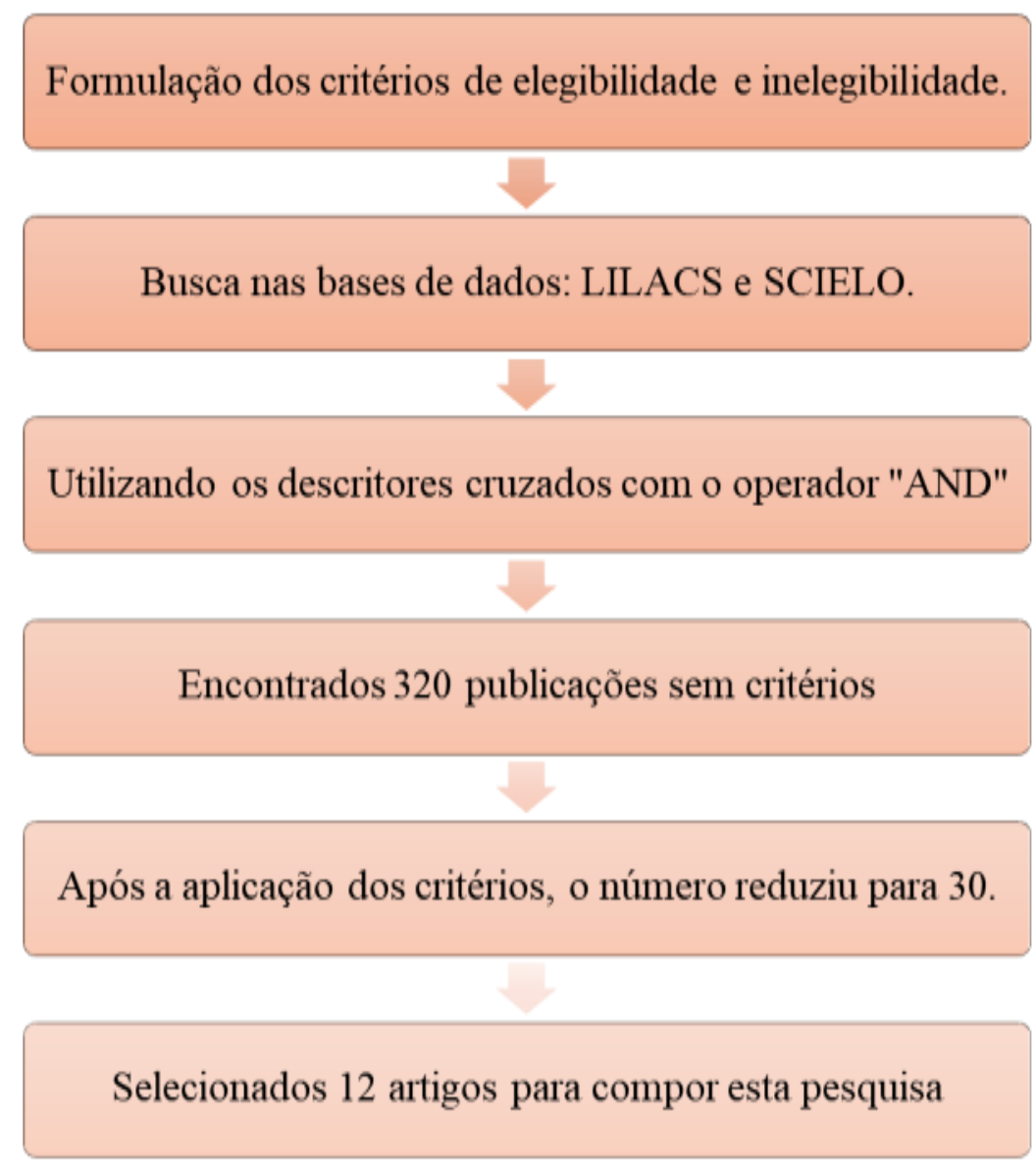

Fonte: Pires, et al. (2021).

As variáveis selecionadas para análise foram: autores, ano que foi realizada a pesquisa, local do estudo, esta análise oriunda dos periódicos permitiu a identificação de 12 artigos, conforme a categoria I e II que caracteriza o conteúdo dos estudos. Perante a pesquisa de artigos realizados observou-se que nos anos de 2012 a 2021 foram encontrados artigos para a complementação do trabalho. Os artigos selecionados para compor esta revisão foram organizados e apresentados através de uma síntese completa dos estudos conforme título, ano, país, autor, base de dados e resultados do estudo, facilitando assim no processo de análise e interpretação dos presentes para compor esta revisão integrativa (Tabela 1) 
Tabela 1: Síntese completa dos estudos selecionados.

\begin{tabular}{|c|c|c|c|c|c|}
\hline & Título & País/ Ano & Autores & $\begin{array}{c}\text { Base de } \\
\text { Dados }\end{array}$ & Resultados \\
\hline 1 & $\begin{array}{l}\text { Assistência de } \\
\text { enfermagem na } \\
\text { prevenção de } \\
\text { infecção de sítio } \\
\text { cirúrgico } \\
\text { perioperatório. }\end{array}$ & $\begin{array}{l}\text { Brasil } \\
2019 \\
\text { Categoria } \\
\quad \text { (II) }\end{array}$ & $\begin{array}{l}\text { Lagemann, } \\
\text { et al., } 2019\end{array}$ & Scielo & $\begin{array}{l}\text { Para a prevenção de ISC nos cuidados perioperatórios é } \\
\text { necessário a educação do paciente/família, banho e curativo } \\
\text { diário no pré e pós-operatório, troca de luvas em todo } \\
\text { atendimento, controle glicêmico, higiene das mãos para a equipe } \\
\text { e acompanhamento pós-alta hospitalar. }\end{array}$ \\
\hline 2 & $\begin{array}{l}\text { Intervenções de } \\
\text { Enfermagem na } \\
\text { prevenção de } \\
\text { infecção de sítio } \\
\text { cirúrgico em } \\
\text { neurocirurgia: } \\
\text { revisão integrativa } \\
\text { da literatura. } \\
\end{array}$ & $\begin{array}{l}\text { Brasil } \\
2014 \\
\text { Categoria } \\
\quad \text { (II) }\end{array}$ & $\begin{array}{l}\text { Abreu, } \\
2014 .\end{array}$ & Scielo & $\begin{array}{l}\text { A enfermagem orienta quando a tricotomia utilizando o } \\
\text { tricotomizador elétrico, assepsia adequada, quanto a importância } \\
\text { do material esterilizado para cirurgia de grande porte e dessa } \\
\text { maneira intervir para a prevenção da ISC. }\end{array}$ \\
\hline 3 & $\begin{array}{l}\text { Intervenções de } \\
\text { enfermagem para } \\
\text { reduzir infecção do } \\
\text { sítio cirúrgico em } \\
\text { cirurgias } \\
\text { potencialmente } \\
\text { contaminadas: } \\
\text { revisão integrativa. }\end{array}$ & $\begin{array}{l}\text { Brasil } \\
2020 \\
\text { Categoria } \\
\quad \text { (II) }\end{array}$ & $\begin{array}{l}\text { Martins, et } \\
\quad \text { al. }\end{array}$ & Lilacs & $\begin{array}{l}\text { As intervenções de enfermagem para aredução de ISC são: } \\
\text { realização de curativos diários, utilização de materiais } \\
\text { esterilizados, verificação do tipo de dreno que a paciente está } \\
\text { utilizando e controle das comorbidades. }\end{array}$ \\
\hline 4 & $\begin{array}{l}\text { Infecção do sítio } \\
\text { cirúrgico: medidas } \\
\text { devigilância e } \\
\text { prevenção risco são } \\
\text { institucionamente } \\
\text { aplicadas? }\end{array}$ & $\begin{array}{l}\text { Brasil } \\
2019 \\
\text { Categoria } \\
\quad \text { (I) }\end{array}$ & $\begin{array}{l}\text { Batista, et } \\
\text { al., } 2019\end{array}$ & Scielo & $\begin{array}{l}\text { As intervenções realizadas para redução de ISC como: banho } \\
\text { prerioperatório e a descolonização e limpeza corporal com } \\
\text { gluconato de clorexidina, tricotomia no local do procedimento a } \\
\text { ser realizado e antibioticoprofilaxia se necessário. }\end{array}$ \\
\hline 5 & $\begin{array}{l}\text { Atuação do } \\
\text { Enfermeiro na } \\
\text { prevenção de } \\
\text { infecção do sítio } \\
\text { cirúrgico. } \\
\end{array}$ & $\begin{array}{l}\text { Brasil } \\
2020 \\
\text { Categoria } \\
\quad \text { (I) }\end{array}$ & $\begin{array}{l}\text { Teles, et } \\
\text { al., } 2020\end{array}$ & Lilacs & $\begin{array}{l}\text { Tais ISC se desenvolvem no local do procedimento cirúrgico, } \\
\text { estes são vítimas de erros que poderiam ser evitados pela } \\
\text { assistência através de intervenções planejadas, como educação } \\
\text { continuada, atualizações em cursos e eventos científicos para os } \\
\text { profissionais atuantes, checagem e ciência do profissional quanto } \\
\text { aos treinamentos realizados pela empresa. }\end{array}$ \\
\hline 6 & $\begin{array}{c}\text { Fatores associados } \\
\text { a infecção de sítio } \\
\text { cirúrgico em um } \\
\text { hospital na } \\
\text { Amazônica } \\
\text { Ocidental } \\
\text { Brasileira. } \\
\end{array}$ & $\begin{array}{l}\text { Brasil } \\
2012 \\
\text { Categoria } \\
\quad \text { (II) }\end{array}$ & $\begin{array}{l}\text { Aguiar, et } \\
\text { al., } 2012\end{array}$ & Scielo & $\begin{array}{l}\text { Os fatores apresentados neste estudo, identificaram que as ISC } \\
\text { apareceram em pacientes que realizaram cirurgias de } \\
\text { colecistectomia e laparotomia e que apresentaram comorbidades } \\
\text { como hipertensão e diabetes, tricotomizados com lâminas de } \\
\text { barbear além de outros. }\end{array}$ \\
\hline 7 & $\begin{array}{l}\text { Fatores de riscopara } \\
\text { infecção de sítio } \\
\text { cirúrgico em } \\
\text { procedimentos } \\
\text { cirúrgicos } \\
\text { cardíacos. } \\
\end{array}$ & $\begin{array}{l}\text { Brasil } \\
2018 \\
\text { Categori } \\
\text { a(II) }\end{array}$ & $\begin{array}{l}\text { Barros, et } \\
\text { al., } 2018\end{array}$ & Scielo & $\begin{array}{l}\text { Os fatores de risco presentes nos procedimentos de cirurgia } \\
\text { cardíaca com alto potencial de ISC inclui: não preparo da pele } \\
\text { antes de incisão cirúrgica, não realizar troca de luva estéril } \\
\text { quando necessário, reutilizar materiais abertos ou com potencial } \\
\text { risco de contaminação externa, como por exemplo: gase estéril e } \\
\text { caneta de bisturi eletrocautério. }\end{array}$ \\
\hline 8 & $\begin{array}{l}\text { Saberes dos } \\
\text { enfermeiros sobre } \\
\text { prevenção de } \\
\text { infecção do sítio } \\
\text { cirúrgico. }\end{array}$ & $\begin{array}{l}\text { Brasil } \\
2020 \\
\text { Categoria } \\
\quad \text { (I) }\end{array}$ & $\begin{array}{l}\text { Souza, et } \\
\text { al., } 2020\end{array}$ & Lilacs & $\begin{array}{l}\text { O risco de infecções em pacientes podem ser evitados através da } \\
\text { adoção de ações preventivas, como a lavagem das mãos, o uso de } \\
\text { epi’s, realização das trocas de curativos diários de forma técnica } \\
\text { e asséptica, além do uso de insumos adequados, conhecimento } \\
\text { técnico científico harmonioso e estímulo do relacionamento } \\
\text { eficaz entre a equipe. }\end{array}$ \\
\hline 9 & $\begin{array}{c}\text { Indicadores } \\
\text { dequalidade em } \\
\text { enfermagem com } \\
\text { ênfase no centro } \\
\text { cirúrgico: revisão } \\
\text { integrativa da } \\
\text { literatura. }\end{array}$ & $\begin{array}{l}\text { Brasil } \\
2017 \\
\text { Categoria } \\
\quad \text { (I) }\end{array}$ & $\begin{array}{l}\text { Baldo, et } \\
\text { al., } 2017\end{array}$ & Lilacs & $\begin{array}{l}\text { Para o gerenciamento de indicadores são feitos } \\
\text { acompanhamentos diários realizados no pré, intra e } \\
\text { transoperatório, como preenchimento do SAEP e Checklist de } \\
\text { cirurgia segura, capazes de minimizar ações negligentes. }\end{array}$ \\
\hline 10 & $\begin{array}{l}\text { A competência } \\
\text { relacional de } \\
\text { enfermeiros em }\end{array}$ & $\begin{array}{c}\text { Brasil } \\
2020 \\
\text { Categoria }\end{array}$ & $\begin{array}{c}\text { Santos, et } \\
\text { al., } 2020\end{array}$ & Lilacs & $\begin{array}{l}\text { O CC dispõe de processos que potencializam o desgaste físico e } \\
\text { mental, portanto a competência relacional é feita através do } \\
\text { gerenciamento de todo o processo assitencial, da comunicação }\end{array}$ \\
\hline
\end{tabular}




\begin{tabular}{|c|c|c|c|c|c|}
\hline \multirow{1}{*}{11} & $\begin{array}{c}\text { unidades de centros } \\
\text { cirúrgicos. }\end{array}$ & (I) & $\begin{array}{c}\text { entre a equipe multidisciplinar, e da gestão de pessoas através do } \\
\text { dimensionamento de escalas e inteligência emocional. }\end{array}$ \\
$\begin{array}{c}\text { Conhecimento dos } \\
\text { profissionais de } \\
\text { enfermagem sobre } \\
\text { fatores de risco } \\
\text { relacionados à } \\
\text { infecção de sítio } \\
\text { cirúrgico. }\end{array}$ & $\begin{array}{c}\text { Brasil } \\
2015 \\
\text { Categoria } \\
\text { (II) }\end{array}$ & $\begin{array}{c}\text { Carvalho, } \\
\text { et al., } \\
12\end{array}$ & 2015 & Scielo & $\begin{array}{l}\text { Todo profissional da área de enfermagem deve possuir domínio } \\
\text { sobre a sua assistência voltada para prevenção e recuperação do } \\
\text { paciente afetado, dessa maneira é necessário que este esteja } \\
\text { emconstante atualização evitando assim arealização e exposição } \\
\text { desse paciente a fatores de risco que predisponham a ISC. }\end{array}$ \\
\hline $\begin{array}{c}\text { Assistência de } \\
\text { enfermagem na } \\
\text { segurança do } \\
\text { paciente cirúrgico: } \\
\text { revisão integrativa. }\end{array}$ & $\begin{array}{c}\text { Brasil } \\
\text { Categoria } \\
\text { (I) }\end{array}$ & $\begin{array}{c}\text { Barros, et } \\
\text { al., 2016 }\end{array}$ & Scielo & $\begin{array}{l}\text { Para manter a segurança do paciente cirúrgico, o enfermeiro } \\
\text { precisa prestar assistência de enfermagem livre de negligências e } \\
\text { utilizar intervenções comfoco no bem-estar do paciente como: } \\
\text { uso de coberturas especiais nos curativos, troca de 24 a 48 horas, } \\
\text { realizar técnica estéril e cuidados com o dreno. }\end{array}$ \\
\hline
\end{tabular}

Fonte: Pires, et al (2021).

\section{Discussão}

\subsection{Categoria I: Gerenciamento do processo de enfermagem para prevenção de ISC.}

No CC, todos da equipe multidisciplinar são dependentes de uma adequada estrutura e disponibilidade de recursos, tais como insumos e suportes assistenciais, tornando assim uma assistência segura sem oferecer riscos de infecções e preservando a integridade dos pacientes, dessa maneira cabe ao enfermeiro a responsabilidade no gerenciamento dessa equipe e dos cuidados assistenciais aos pacientes que ali se encontram, zelando por sua segurança, qualidade de atendimento e recuperação (Martins, et al., 2016).

Segundo Batista et al. (2019) e Baldo et al. (2017), o gerenciamento dedados cirúrgicos oriundos de indicadores de avaliação são muito importantes, visto que os critérios precisos de vigilância para o controle da ISC permitem que sejam realizadas prevenções rotineiras com o paciente no pré e transoperatório melhorando assim, a qualidade assistencial.

Segundo OMS (2016), as ISCs consistem em um dos eventos resultados da insuficiência da assistência de enfermagem, visto que se caracterizam mundialmente como deficiência nos parâmetros de controle da qualidade voltados para o serviço prestado ao paciente pelo hospital.

Segundo Santos et al. (2020) e Barros et al. (2016), o centro cirúrgico demanda de atividades que potencializam o desgaste físico e mental do profissional, através de atividades administrativas e assistenciais, portanto o gerenciamento está inteiramente ligado a assistência aos pacientes cirúrgicos, portanto, esta atividade é realizada como forma de acompanhamento da assistência operatória, visto que os indicadores supracitados demandam para que sejam realizadas mudanças e melhorias na forma de assistir o paciente cirúrgico e se evitar a ISC.

No serviço de saúde faz-se necessário a comunicação em todo o processo de assistencial, onde a ausência desse mecanismo pode estar relacionada à abordagem punitiva dos erros. Assim, é importante enfatizar a necessidade da implementação de um processo organizacional que permita à equipe multidisciplinar identificarem e explicitarem os erros cometidos e suas causas, permitindo a criação de estratégias de segurança do paciente com a finalidade de prevenção de possíveis complicações (Abreu, et al., 2019).

Os indicadores utilizados no centro cirúrgico para a segurança do paciente e checklist de cirurgia segura são: A Sistematização da Assistência de Enfermagem Perioperatória (SAEP) juntamente com a Visita Pré-Operatória de Enfermagem (VPOE) possibilita ao enfermeiro conhecer o seu cliente antes de todo o processo, levando a traçar planejamentos de cuidados e melhores resultados (Jost, et al., 2018).

A Sistematização da Assistência de Enfermagem Perioperatória (SAEP) somada com a Visita Pré-Operatória de Enfermagem possibilita ao enfermeiro conhecer o seu cliente antes de todo o processo, levando a traçar planejamentos de 
cuidados e melhores resultados. O SAEP faz parte da sistematização voltada para o cuidar integral e igualitário ao paciente, permitindo assistência direta ao indivíduo, seu problema e estado de saúde (Amaral, et al., 2017).

Neste Sentido, Teles et al. (2020) e Souza et al. (2020), referem que é muito importante o saber do profissional enfermeiro, visto que desde a simples lavagem das mãos na técnica correta, informação na assepsia que esse paciente necessita realizar, requer a prevenção e segurança desse paciente, portanto o gerenciamento do enfermeiro com tais questões é prioritariamente importante.

Sendo assim, cabe à equipe de enfermagem, acolher e auxiliar o paciente no período perioperatório através da sistematização da assistência de enfermagem, e a implementação do processo de enfermagem, no qual é possível iniciar as intervenções para todo e qualquer paciente. A execução dessas intervenções de enfermagem é realizada com foco nas medidas preventivas, assim como sua implementação de maneira adequada, reduzindo a SC (Almeida, 2015).

Considerando a importância na responsabilidade pelos cuidados diretamente ligados ao paciente e com a ferida cirúrgica, a enfermagem vivencia intensa preocupação com a qualidade da assistência, sendo assim, o enfermeiro tende a observar todas as necessidades dos mecanismos de cuidados, sejam eles profiláticos ou curativos, com o intuído de buscar estratégias que visam na qualidade da assistência e na prevenção de ocorrência de ISC (Boaventura, et al., 2019).

Neste contexto, as ações de gerenciamento do processo de enfermagem, está associado à realização de forma otimizada com a Sistematização da Assistência de Enfermagem, dentro de um contexto perioperatório, através da implementação do processo de enfermagem com o qual é possível estabeleceras intervenções de enfermagem e os controles da qualidade do serviço prestado por uma instituição hospitalar.

\subsection{Categoria II: Ações voltadas para a segurança do paciente e redução de ISC}

Segundo Lagemann et al. (2019) e Carvalho et al (2015), a enfermagem é a classe profissional que passa o maior período do tempo com o paciente internado, portanto, assistência de enfermagem ao paciente cirúrgico deve ser voltada para a prevenção de infecção do sítio cirúrgico na fase perioperatória, de maneira que este paciente esteja seguro no pré, intra e pósoperatório com intervenções de enfermagem sendo realizadas beira/leito com o paciente.

O enfermeiro tem por fundamental conduta, desenvolver ações de cuidados individualizados junto à cada paciente, se direcionando para um processo assistencial e profissional, que deve ser traçado no conhecimento técnico e científico, através de competências assistenciais em geral e na relação interpessoal entre equipe, paciente e família para que possa favorecer ao cliente uma criação de um ambiente humanizado e seguro (Santos, et al., 2020).

Para Cláudia et al. (2014) e Martins et al. (2020), intervenções de enfermagem estão associadas a assepsia e antissepsia realizadas no transoperatório pelo profissional, sendo estas medidas capazes de evitar e reduzir a ISC e reduzir as cirurgias potencialmente contaminadas, desta maneira este profissional consegue ofertar segurança ao paciente e ao mesmo tempo a sua equipe, prevenindo assim a IST oriunda dos próprios profissionais devido ao manuseio ofertado.

Segundo Figueiredo (2012), as Cirurgias Potencialmente Contaminadas envolvem cirurgias realizadas em tecidos que possuem flora residente não numerosa ou onde a descontaminação é um processo difícil, sendo assim já ocorreu a abertura do sistema respiratório, geniturinário ou digestório, sem contaminação significativa de infecção.

A enfermagem é a classe responsável pela prevenção e controle das infecções, dessa maneira suas ações são dependentes e relacionadas. Uma das mais importantes causas de infecções, é a que ocorre de forma cruzada, sendo esta ocasionada pela transmissão de um microrganismo que é levado de um paciente para o outro, ou também através das mãos dos profissionais, acompanhantes e visitantes. Perante os procedimentos de prevenção de infecções cruzadas, evidencia-se que a lavagem das mãos é eficaz na prevenção das infecções hospitalares (Henriques, et al., 2016). 
Para Aguiar et al. (2012) e Barros et al. (2018), os fatores associados a ISC referente a procedimentos cirúrgicos, requer dados precisos oriundos dos pacientes e dos procedimentos realizados na hora e momento da cirurgia, de modo que avalie a segurança do paciente antes ou durante todo e qualquer procedimento.

Com o avanço do conhecimento científico, medidas têm sido preconizadas por organizações internacionais e nacionais a fim de controlar a propagação de microrganismos. Dentre elas destacam-se: a adesão de profissionais de saúde às medidas como higienização das mãos, o uso de equipamentos de proteção individual e a identificação de precauções relativas aos pacientes colonizados por microrganismos resistentes (Oliveira, et al., 2015).

Segundo Oliveira et al. (2018) diagnóstico da ISC, dá-se com a metodologia oriunda do (Sistema Nacional de Vigilância de Infecções Hospitalares - NNISS), quer e força que a infecção pode acontecer em 30 dias após a data da cirurgia ou, em caso de prótese, em até um ano após o procedimento. Os fatores de risco para desenvolver ISC são: idade, presença de doenças crônicas, estado nutricional, imunossupressão, tabagismo, natureza e local da cirurgia, tempo de internação pré e pósoperatório, grau de contaminação da cirurgia e infecções existentes.

Portanto, destaca-se a importância do enfermeiro para o desenvolvimento de ações que visem amenizar o índice de eventos adversos que ocorrem no centro cirúrgico, atuando de forma dinâmica e decisiva no controle e prevenção de qualquer risco, evitando possíveis agravos relacionados à ISC (Souza, 2020).

Nesse contexto, a assistência de enfermagem voltada para a segurança do paciente, inclui cuidados rigorosos no pósoperatório, visto que o acompanhamento durante a internação hospitalar desse paciente permite resultados significativos no controle e na redução de taxas de infecção, diminuindo o risco de subnotificações e infecções hospitalares.

\section{Considerações Finais}

A ISC é, hoje uma das principais complicações oriundas pós procedimento cirúrgico, podendo resultar em morbidade, mortalidade e altos custos hospitalares. Dessa maneira, as ações preventivas realizadas pela enfermagem para reduzir as ISC são tomadas de ações imprescindíveis.

Com o estudo pode-se perceber que o profissional enfermeiro e a sua equipe são os profissionais de saúde que estão em constante troca e contato direto com o paciente, e tais profissionais são dotados de conhecimento técnico-científico para avaliar e prestar assistência adequada, de forma que atenda cada paciente conforme o grau de necessidade, visando prevenir a ocorrência de complicações no pós-cirúrgico imediato e mediato.

Sendo assim, evidencia-se a necessidade de realizar novos estudos embasados em evidências mais fortes, para identificação de fatores de risco relacionados às ISC, sendo que estas podem trazer implicações diretas para a prática de enfermagem.

\section{Referências}

Abreu, I. M., Rocha, R. C., Avelino, F. V. S. D., Guimarães, D. B. O. Nogueira, L. T., \& Madeira, M. Z. A. (2019). Cultura de segurança do paciente em centro cirúrgico: visão da enfermagem. Rev Gaúcha Enferm. 40(esp): e20180198.

Almeida, M. C. (2015). Enfermagem perioperatória e sua inserção nos cursos de graduação. Dissertação (Mestrado em enfermagem): Universidade Federal do Amazonas, Manaus.

Amaral, J. A. B., Spiri, W. C., \& Bocchi, S. C. M. (2017). Indicadores de Qualidade em Enfermagem com Ênfase no Centro Cirúrgico. Rev. SOBECC, 22(1): $42-51$.

Batista, L. B. L., Silva, S. B. A., Martins, D. A., Lara, M. O., \& Lucas, T. C. (2019). Infecção do sítio cirúrgico: medidas de vigilância e prevenção de risco são institucionalmente aplicadas? Cogitare Enfermagem, 24. http://dx.doi.org/10.5380/ce.v24i0.62968.

Boaventura, J. E. M., Cordeiro, A. L. A. O., Barros, C. S. M. A., Moreira, B. S. G., Lobo, J. O., \& Pedreira, L. C. (2020). Infecções de sítio cirúrgico: incidência e perfil de resistência antimicrobiana em unidade de terapia intensiva. Revista Baiana De Enfermagem. https://doi.org/10.18471/rbe.v33.33595 
Brasil, (2017). Agência Nacional de Vigilância Sanitária. Critérios Diagnósticos de Infecções Relacionadas à Assistência à Saúde/Agência Nacional de Vigilância Sanitária. Brasília: Anvisa.

Figueiredo, E. G., Balasso, G. T., \& Teixeira, M. J. (2012). Infecções em pós-craniotomias: revisão literária. Arquivos brasileiros de neurocirurgia, 31 (4), 219-223. doi:10.1055/s-0038-1625741.

Franco L. M. C., Almeida A. G. I., Duarte G. M. H., Lamounier L., Pinto T. S., Pereira P. F. S., Chianca T. C. M., \& Ercole F. F. (2017). Efeitos do banho préoperatório na prevenção de infecção cirúrgica: estudo clínico piloto. REME - Rev Min Enferm. 21, e1053. 10.5935/1415-2762.20170063.

Griep, R. H., da Fonseca, M. J. M., Melo, E. C. P., Portela, L. F., \& Rotenberg, L. (2013) Enfermeiros dos grandes hospitais públicos no Rio de Janeiro: características sociodemográficas e relacionadas ao trabalho. Rev. Bras. Enferm., 66, 151-157.

Henriques, A. H. B., Costa, S. S., \& Lacerda, J. S. (2016). Assistência de Enfermagem na Segurança do Paciente Cirúrgico: Revisão Integrativa. Cogitare Enferm. Out/dez, 21(4): 01-09.

Jost, M. T., Viegas, K., \& Coregnato, R. C. A. (2018). Sistematização da assistência de enfermagem ISCoperatória: revisão integrativa. Rev. Sobecc, São Paulo. 23(4): 218-225.

Martinz, F. Z., \& Dall'agnol, C. M. (2016). Centro cirúrgico: desafios e estratégias do enfermeiro nas atividades gerenciais. Rev Gaúcha Enferm. dez, 37(4), e56945.

Oliveira, A. C., \& Gama, C. S. (2015). Avaliação da adesão às medidas para a prevenção de infecções do sítio cirúrgico pela equipe cirúrgica. Rev. esc. enferm. USP. 49(5), 767-774.

Oliveira, M. C. B., Korb, A., Zocche, D. A. A., Cabral, D. B., Pertille, F., Frigo, J. (2018). Adesão do cheklist cirúrgico à luz da cultura de segurança do paciente. Rev. SOBECC, 23(1), 36-42.

Organização Mundial da Saúde. (2016). Diretrizes globais para a prevenção de infecção do sítio cirúrgico. Organização Mundial da Saúde.

Rocha, J. P., Jardim, L., Clarice, A. S. (2016). O Enfermeiro e a prevenção das infecções do sítio cirúrgico. Cadernos UniFOA, (30), 117-128.

Sandes, S. M. S., Costa, M. F., Santos, G. V., Freitas, L. P., Vasconcelos, A. C. P., Silva, L. S. L. (2019). Lesões provenientes de procedimento cirúrgico: fatores relacionados. Rev. Sobecc, 24(3), 161-167.

Santos, P. V. F., Jesus, K. B., Santana, K. I. S. P., Nogueira, E. C., Cariri, L. S., \& Brito, F. P. G. (2017). infecção do sítio cirúrgico em pacientes no pósoperatório de cirurgias ortopédicas eletivas. Interfaces Científicas - Saúde E Ambiente, 5(2), 71-79. https://doi.org/10.17564/2316-3798.2017v5n2p71-79

Souza, K. V., Serrano, S. Q. (2020). Saberes dos Enfermeiros Sobre Prevenção de Infecção do Sítio Cirúrgico. Rev. Sobecc, 25(1): 11-16.

Souza, I. S. B., Santana, A. C., \& D’alfonso Júnior G. (2018). A ocorrência de infecção do sítio cirúrgico: um estudo de revisão. Rev Med Minas Gerais, 28 (Suppl5): e-S280521.

Santos, D. J., Henriques, S. H., Leal, L. A., Soares, M. I., Chaves, L. D. P., \& Silva, B. R. (2020). A Competência Relacional de Enfermeiros de Centro Cirúrgico. Rev. Enferm. Uerj, 28: e51314.

Severo, E. (2016). Ações de enfermagem na prevenção de infecções hospitalares: Uma revisão integrativa da literatura. Faculdade Método de São Paulo, from https://www.ccih.med.br/acoes-de-enfermagem-na-prevencao-de-infeccoes-hospitalares-uma-revisao-integrativa-da-literatura/

Shi, Z., Tang, S., Chen Y., Lee, D. T., Chair, S. Y., Jiang, B., Zhu, X., Pan, X., Yang, J., \& Qin, Y. (2014). Application of a glycaemic control optimization program me in patients with stress hyperglycaemia. British Association of Critical Care, 21 (5): 304-310.

Souza, K. V., \& Serrano, S. Q. (2020). Saberes dos Enfermeiros Sobre Prevenção de Infecção do Sítio Cirúrgico. Rev. Sobecc, 25(1): 11-16. 